\title{
Zirkel und Disparitäten - Das Paradox der wissenschaftlichen Bildung
}

\section{Karsten Kenklies}

Vorbemerkungen

Wissenschaftliche Bildung - welch` hehres Wort. Wohl kaum jemand würde bestreiten, daß es sich hier nicht nur um eine sinnvolle, sondern gar um eine notwendige Sache handelt.

Kommen hier doch zwei Begriffe zusammen, die wie kaum andere eine Aura der Erhabenheit und Positivität aufweisen. Zusammengenommen verbreiten sie dann auch einen Glanz, dem sich kaum jemand zu entziehen vermag. Und mit dem sich so manche_r wohl auch gerne schmückt in Zeiten, in denen Glanz relevanter zu sein scheint als die Substanz, von der jener Glanz (leider nur vermeintlich) ausgeht. Und das allemal mit Blick auf jene beiden Phänomene, auf welche sich dieses strahlende Wort der Wissenschaftlichen Bildung bezieht: Wissenschaft und Bildung.

Nun ist es heute und hier nicht meine Aufgabe, über den vermeintlichen oder wahrhaftigen Glanz von Wissenschaft und Bildung jenseits und diesseits von Student Assessment, BolognaReform und Drittmittel-Wahn zu sinnieren. Die Frage wird vielmehr sein, ob es ein Konzept Wissenschaftlicher Bildung überhaupt geben kann - und ob es dieses geben kann in einer Form, die es anschlußfähig macht für das, was hierzulande Wissenschaft genannt wird. Denn tatsächlich muß sich ja jedes Konzept Wissenschaftlicher Bildung dem Anspruch stellen, für jede_n Wissenschaftler_In zugänglich, d.h. nachvollziehbar und einsichtig zu sein. Denn schließlich handelt es sich letztlich um ein normativ besetztes Konzept, mit dem bestimmte Erwartungen an Wissenschaftler_Innen verbunden sind - Erwartungen, von denen nun noch nicht gänzlich festgelegt ist, ob man sich ihnen freiwillig unterwerfen darf oder doch sollte oder gar schon soll.

Wie bereits deutlich wurde, soll der Begriff der Wissenschaftlichen Bildung hier in spezifischer Weise verwendet werden: auf die Weise, wie er in dem vorliegenden Band verstanden wird. Wissenschaftliche Bildung ist demnach eine spezifische Form der Bildung für Wissenschaftler_Innen - und nicht der Teilbereich von Wissensbeständen, der aus (für alle angeblich lebensnotwendigen) (natur-)wissenschaftlichen Grundkenntnissen bestehen würde. ${ }^{1}$ Derartige Diskussionen gab und gibt es natürlich. Erinnert sei an die Invektive von Ernst Peter

\footnotetext{
${ }^{1}$ An dieser Stelle auch noch daran erinnernd, daß die Assoziation von Bildung mit deklarativen Wissensbeständen generell eine besonders kurzsichtige Idee von Bildung darstellt - und sich eine Idee von Allgemeinbildung in der Moderne eben nicht wirklich überzeugend darstellen läßt als Addition geisteswissenschaftlicher und naturwissenschaftlicher deklarativer Wissensbestände.
} 
Fischer gegen das Buch Bildung ${ }^{2}$ von Dietrich Schwanitz, dessen Kompendium angeblich nur Wissensbestände der Geistes- und Sozialwissenschaften enthielte und daher ergänzt werden müßte durch naturwissenschaftliches Basiswissen, ergänzend vorgelegt von Fischer in Die andere Bildung - Was man von den Naturwissenschaften wissen sollte $e^{3}$. Das soll hier nicht unser Problem sein, sondern die Frage danach, ob sich sinnvoll eine Idee von Bildung formulieren ließe, die gesondert und ausschließlich für Wissenschaftler_Innen relevant wäre und daher den Namen Wissenschaftliche Bildung verdienen würde - etwa in Absetzung zu Allgemeinbildung.

Was genau steckt demnach hinter der Frage nach der Wissenschaftlichen Bildung? Offensichtlich ließen sich verschiedene Konzepte Wissenschaftlicher Bildung denken differenziert durch ihren jeweiligen Begriff der Bildung, der sich am Phänomen/ Begriff der Wissenschaft abarbeitet. Dieser muß denn auch konstant gehalten werden mit Blick auf verschiedene Ideen von Wissenschaftlicher Bildung, insofern sich diese Konzepte eben an alle Wissenschaftler_Innen richten und daher Klarheit und Konsens darüber herrschen sollte, an wen sich solche Überlegungen überhaupt richten (wer die Betroffenen sind). Während also der Begriff der Bildung je nach Konzept variieren kann, kann es der den Bildungsbegriff qualifizierende Begriff der Wissenschaft(lichkeit) nicht. Was mit anderen Worten bedeutet: Ein Konzept Wissenschaftlicher Bildung steht und fällt einerseits mit der Überzeugungskraft seines spezifischen Bildungsverständnisses, und andererseits mit der Möglichkeit der Formulierung einer Idee DER Wissenschaft. Dieses wäre auch insofern nötig, als daß sich Wissenschaftliche Bildung eben von anderer Bildung unterscheiden müßte, will sie sich als spezielle Form von Bildung behaupten.

Im Folgenden soll es nicht um die Diskussion des besonderen Bildungsbegriffs gehen, der in diesem Band eventuell vorgestellt und debattiert wird. Meine Frage wird sein: Gibt es einen Gegenstand Wissenschaft, der einheitlich und spezifisch genug wäre, um als qualifizierendes Adjektiv verwendet werden zu können; oder anders: Was könnte wissenschaftlich bedeuten, und hat dieses Adjektiv eine einigermaßen stabile Bedeutung? Die Antwort auf diese Frage wird „Nein“ lauten: Die Voraussetzung der Einheit der Wissenschaft scheitert auf mindestens drei Ebenen: 1) auf der Ebene der Idee von der Einheit der Wissenschaft; 2) auf der Ebene Praxis der Wissenschaften im Vergleich zueinander; und 3) auf der Ebene der einzelnen Wissenschaften selbst und dem Verhältnis der Wissenschaftler_Innen zu ihrer Tätigkeit, dem

\footnotetext{
${ }^{2}$ Schwanitz, Dietrich: Bildung. Alles was man wissen muß, Frankfurt am Main: Eichborn 1999.

${ }^{3}$ Fischer, Ernst Peter: Die andere Bildung - Was man von den Naturwissenschaften wissen sollte, München: Ullstein 2001.
} 
Betreiben von Wissenschaft. Dieses und die Folgen dieser dreifachen Uneinheitlichkeit für ein Konzept Wissenschaftlicher Bildung sollen nachfolgend näher erläutert werden.

1) Die Uneinheitlichkeit der Ideen von der Einheit der Wissenschaft

Die Vorstellung, daß es einen Begriff von Wissenschaft gibt, der alle vorkommenden Einzelwissenschaften umfaßt, ist intuitiv sicher ebenso einsichtig, wie sie irreführend ist. Sie wurde wohl genährt durch eine spezifische Theorie der Bedeutung, die besagt, daß Begriffe gewissermaßen eine Kernreferenz haben, welche alle vorkommenden Signifikate umfaßt (vorgebildet in der platonischen Theorie der Idee). Dazu tritt eine vage Vorstellung, nach der sich die Genese der modernen Wissenschaften als Prozeß der Ausdifferenzierung aus einer Einheitswissenschaft vollzog und sich auch weiter vollzieht. Weder das eine, noch das andere scheint - bei genauerer Betrachtung - richtig zu sein. Tatsächlich mag mit Blick auf die verschiedenen Wissenschaften so etwas wie eine Familienähnlichkeit bestehen (ein Begriff, den Wittgenstein einführte als Grundlage einer alternativen Bedeutungstheorie ${ }^{4}$ ); und bereits ein kurzer Blick auf die Geschichte der Wissenschaften, dargestellt etwa von Serres ${ }^{5}$, zeigt, daß die Vorstellung einer umfassenden Ausdifferenzierungsgenese der Einzelwissenschaften nicht wirklich haltbar ist: Es gab verschiedene Herkünfte, unterschiedliche Wissenstraditionen und Wissenspraktiken, die zusammenflossen in das, was wir heute so scheinbar einheitlich Wissenschaft nennen.

Es überrascht denn auch kaum, daß bereits die Vorstellung davon, worin die Einheit der Wissenschaften besteht, keineswegs einheitlich ist - trotz oder gerade weil die Sehnsucht, Dinge zu vereinheitlichen oder als Einheit wahrzunehmen, so groß ist, daß jeder Weg recht zu sein scheint, diese Behauptung einer Einheit zu stützen und zu verteidigen. ${ }^{6}$

Bereits auf einer noch recht abstrakten Ebene läßt sich fragen, ob mit Einheit tatsächliche Einheitlichkeit, d.h. Eins-Sein (unum esse), gemeint sei (etwa wie die anscheinend zwei Seiten eines Moebius-Bandes, die letztlich eben doch ein und dieselbe Seite sind), oder so etwas wie die Harmonie ansonsten disparater Einheiten (etwa wie ein Puzzle, in welchem grundverschiedene Teile zusammen ein sinnvolles Ganzes ergeben). Wie Hacking zeigen kann, basieren manche Theorien der Einheit der Wissenschaften auf der ersten, andere auf der zweiten Vorstellung. Darüber hinaus lassen sich diese Theorien auch auf andere Weise mit

\footnotetext{
${ }^{4}$ Wittgenstein, Ludwig: Philosophische Untersuchungen, Frankfurt am Main: Suhrkamp 1953.

${ }^{5}$ Serres, Michel (Hrsg.): Éléments d'histoire des sciences, Paris: Bordas 1989 (deutsch: Elemente einer Geschichte der Wissenschaften, Frankfurt: Suhrkamp 1998).

${ }^{6}$ Die folgenden Ausführungen basieren hauptsächlich auf: Hacking, Ian: The Disunities of the Sciences, in: Galison, Peter/ Stump, David J. (Hrsg.): The Disunity of Science: Boundaries, Contexts, and Power, Stanford: University Press 1996, S. 37-74.
} 
Blick auf ihre Einheitsvorstellung einteilen: a) metaphysische, b) praktische, und c) logische Einheitspostulate. Eine metaphysische Gründung der Einheitsidee wird, so Hacking, etwa durch den Glauben: „Es gibt eine wissenschaftliche Welt, eine Realität, eine Wahrheit!“ gesetzt. Hieraus hat man etwa gefolgert, daß die Dinge in wissenschaftlicher Perspektive miteinander verbunden sind; für manche sind sie dann auch noch etwa in der Einen Großen Theorie beschreibbar (etwa in Form eines Reduktionismus, d.h. der Rückführung aller Theorien auf eine einzelne Theorie oder der Übersetzung alle wissenschaftlichen Theorien in den Jargon einer einzelnen wissenschaftlichen Disziplin). Eine andere Version dieser Idee wäre die proportional identische Strukturierung der Welt auf allen Ebenen (eine Neuauflage der alten Idee der Parallelität von Mikro- und Makrokosmos, z.B. als Vorstellung, daß alles trinitarisch oder - in einer modernen Version - als Fraktal, z.B. als Mandelbrot-Menge, geordnet ist). Solche Grundlegungen erzeugen dann auch unterschiedliche Erkenntnispraktiken: So suchen die Verbundenheitstheoretiker_Innen eben nach solchen Verbindungen - und das wäre die generelle Charakteristik wissenschaftlichen Arbeitens -, während die Anhänger_Innen der Großen Theorie ihre Arbeit als Wissenschaftler_Innen generell als Reduktionsarbeit betrachten und die Parallelitätsbefürworter_Innen einem Strukturalismus das Wort reden (d.h. sie suchen genau jene überall immer wieder auftauchenden Strukturen).

Die eher auf der praktischen Ebene angesiedelten Einheitsideen sehen erst (oder allein) bei der Frage nach der spezifischen Rationalität wissenschaftlichen Arbeitens jene Einheitlichkeit realisiert. So etwa im angeblich universal-wissenschaftlichen Tun der Hypothesenbildung und -überprüfung (Falsifikation), oder gar in einer universal angewendeten konkreten Methode. Die Verfechter_Innen einer logischen Einheitsidee sehen vor allem ein einheitliches Sprachkalkül als grundlegende Einheit der Wissenschaften an. Sie thematisieren etwa den angeblichen Unterschied von wissenschaftlichen und unwissenschaftlichen Sätzen, von sinnvollen und sinnlosen Äußerungen und von echten und Scheinproblemen.

Diese nur sehr kurze und geraffte Übersicht über die verschiedenen Wege, auf denen die Einheit der Wissenschaften (zumeist normativ, und am ehesten von Wissenschaftsphilosoph_Innen) konstruiert wird, müßte nun noch entscheidend modifiziert werden für den deutschen Kontext, insofern Hacking (wie die meisten angelsächsischen Wissenschaftsphilosoph_Innen und -historiker_Innen) lediglich den Kontext der sciences vor Augen hat - also das, was im deutschen Kontext mit Naturwissenschaften bezeichnet wird. Mit Blick auf den deutschen Kontext und seinen wesentlich breiteren Wissenschaftsbegriff, 
der die Natur-, aber natürlich auch die Geistes-, Sozial- und etwa die Strukturwissenschaften umfaßt, dürfte das Ausmaß an Pluralität noch weiter zunehmen.

Zu ähnlichen Ergebnissen führt der eher deskriptive Blick auf die realen Abläufe wissenschaftlichen Tuns, also die gelebte Wissenschaft. Mögen auch manche Wissenschaftler_Innen in ihrem Tun von einer Einheitsvision begleitet oder gar gelenkt werden, so zeigen sich doch in der Perspektive auf mehrere Wissenschaften Unterschiede, die so groß sind, daß kaum von einer Einheitlichkeit der realen Wissenschaft gesprochen werden kann.

2) Die Uneinheitlichkeit der Praxis der Wissenschaften

Wie der Blick auf verschiedene theoretische Konzeptionen der Einheit der Wissenschaft zeigt, waren (und sind) es zumeist Wissenschaftsphilosoph_Innen, die eine solche Einheitsvision vertreten. Dabei entspringt diese Vision oft nicht deskriptiven Zugängen, sondern präskriptiven Wissenschaftstheorien. Es waren dann die Wissenschaftshistoriker_Innen und soziolog-Innen, die sich den realen Abläufen von als Wissenschaft klassifizierten Prozessen widmeten und frappierende Diskrepanzen entdeckten zwischen der Idealität und der Realität wissenschaftlichen Arbeitens.

Bereits Ludwig Fleck zeigt 1935 auf, daß Wissenschaften historisch kontextualisiert zu betrachten sind und daß ihnen gewisse Denkstile eigen sind, die von Denkkollektiven repräsentiert werden: Nicht zwangsläufig wird eine Wissenschaft von einem Kollektiv und ein em Stil beherrscht, und schon gar nicht die verschiedenen Wissenschaften. ${ }^{7}$ Die dann seit den 1960er Jahren einsetzende intensive wissenschaftssoziologische und -historische Forschung nahm die seinerzeit völlig ignorierten Erkenntnisse Flecks auf und entwickelte sie weiter. Vorreiter hier waren etwa Thomas S. Kuhn und Paul Feyerabend. Während Kuhn den Begriff des Paradigmas einführte zur Beschreibung realer Denk-und Praxis-Kontexte, in welche man als Wissenschaftler_In einsozialisiert wird, ${ }^{8}$ widmete sich Feyerabend den wissenschaftlichen Erkenntnispraktiken der gefeierten Klassiker und erklärte sie zu Ketzern in Bezug auf eine angeblich universale Wissenschaftsrationalität: Sie erzielten ihre Ergebnisse auf höchst unorthodoxe Weise - eine Beobachtung, die Feyerabend mit der vielfach zitierten

\footnotetext{
${ }^{7}$ Fleck, Ludwig: Entstehung und Entwicklung einer wissenschaftlichen Tatsache. Einführung in die Lehre vom Denkstil und Denkkollektiv [1935], Frankfurt am Main: Suhrkamp 1980.

${ }^{8}$ Kuhn, Thomas S.: The Structure of Scientific Revolutions [1962], Chicago: University of Chicago Press ${ }^{2} 1970$ (deutsch: Die Struktur wissenschaftlicher Revolutionen, Frankfurt am Main: Suhrkamp ${ }^{2} 1976$ ).
} 
(und zumeist als Verteidigung eines völligen Relativismus‘ mißverstandenen) Formel: „Anything goes!“‘ auf den Punkt brachte. ${ }^{9}$

Diese Ergebnisse wurden bestätigt und noch weiter vertieft in den ethnographisch verfahrenden Laboratory Studies, wie sie etwa in den Studien Karin Knorr-Cetinas vorliegen. ${ }^{10}$ Sie befand, daß Wissenschaftsdisziplinen als Epistemic Cultures ${ }^{11}$ anzusehen seien. Der Begriff Epistemic culture „refers to those sets of practices, arrangements and mechanisms, bound together by necessity, affinity and historical coincidence which, in a given area of professional expertise, make up how we know what we know. Epistemic cultures are cultures of creating and warranting knowledge. "12 Ihre Untersuchungen der laborwissenschaftlichen Arbeit von Molekularbiologen und Hochenergie-Physikern machten diese unterschiedlichen Epistemic cultures sichtbar: Es läßt sich nicht behaupten, daß hier, in diesen beiden Laboren, in irgendeiner Weise dasselbe abläuft: „The notion of an epistemic culture takes up where this assessment leaves off. It brings into focus the content of the different knowledge-oriented lifeworlds, the different meanings of the empirical, specific constructions of the referent (the objects of knowledge), particular ontologies of instruments, specific models of epistemic subjects. Epistemic unity, then, is a casualty of the cultural approach to knowledge production." ${ }^{\text {"13 }}$

Und auch hier gilt: Die Untersuchungen bezogen sich allein auf die sciences, d.h. das, was man im Deutschen mit Naturwissenschaften bezeichnet. Unter Einbeziehung der Geistes- und Strukturwissenschaften ist die These einer Einheitlichkeit der Wissenschaften nun gar nicht mehr überzeugend. Bestätigt wird das für den deutschen Kontext etwa durch die Untersuchungen des Heureka Projektes. ${ }^{14}$ Innerhalb dieses Projektes wurden verschiedene Wissenschaftler_Innen zu diversen Aspekten ihrer Arbeit befragt (Formen von Thesen, Strategien und Kriterien zum Stützen oder Falsifizieren von Thesen, Datenerhebungs- und Auswertungsprozesse etc.) - mit dem Ergebnis: „Zum einen sind die Konventionen, mit denen Wissenschaftler anderen von einem Gedanken, einer Annahme, einer Theorie oder einem Modell überzeugen wollen, in den einzelnen Disziplinen sehr unterschiedlich, zum

\footnotetext{
${ }^{9}$ Feyerabend, Paul: Against Method [1975], London: Verso ${ }^{3} 1993$ (deutsch: Wider den Methodenzwang, Frankfurt am Main: Suhrkamp ${ }^{2} 1986$ ).

${ }^{10}$ Etwa: Knorr-Cetina, Karin: The Manufacture of Knowledge. An Essay on the Constructivist and Contextual Nature of Science, Oxford: Pergamon Press 1981 (deutsch: Die Fabrikation von Erkenntnis. Zur Anthropologie der Naturwissenschaft, Frankfurt am Main: Suhrkamp 1984, erweiterte Neuauflage 2002).; Knorr-Cetina, Karin: Epistemic Cultures. How the Sciences Make Knowledge [1999], Cambridge (Mass.): Harvard University Press ${ }^{3} 2003$ (deutsch: Wissenskulturen. Ein Vergleich naturwissenschaftlicher Wissensformen, Frankfurt am Main: Suhrkamp 2002).

${ }^{11}$ Knorr-Cetina, Epistemic Cultures.

${ }^{12}$ Knorr-Cetina, Knowledge Cultures, S. 363.

${ }^{13}$ Knorr-Cetina, Knowledge Cultures, S. 364.

${ }^{14} \mathrm{Vgl}$. auch den Beitrag in diesem Band.
} 
anderen sind sie es aber auch innerhalb einer Disziplin, je nach Ausrichtung der dort Forschenden und je nachdem, ob die Rezipienten des Gedachten mündlich oder schriftlich überzeugt werden sollen. Hinzu kommt, daß sich die Überzeugungskulturen innerhalb der Disziplinen im Laufe der Zeit auch wandeln.“15

Die viel beschworene Einheit der Wissenschaften gibt es nicht: Es gibt viele verschiedene Wissenschaften, die aufgrund genetischer Filiationen, historisch gewachsener institutioneller Kontinuitäten und Familienähnlichkeiten aneinander gekoppelt sind. Und es ist lediglich diese institutionelle Kopplung, und die hiermit oft verbundene Finanzierung, die diese verschiedenen menschlichen Tätigkeiten in einen gemeinsamen Rahmen der Abhängigkeit (und damit der Interessen und Bedürfnisse) stellt - obwohl auch schon das nicht mehr für alle gilt, wenn man sich die völlig unterschiedlichen Situationen etwa von Universitäten und Forschungsinstituten (z.B. der Max-Planck- oder der Helmholtz-Institute) anschaut.

3) Die interne Uneinheitlichkeit der Wissenschaften

Weder der Blick auf die Einheit(lichkeit)sthese selbst, noch der auf das Verhältnis der verschiedenen Wissenschaftsdisziplinen zueinander ließ genug Einheitlichkeit erkennen, um dem Begriff der Wissenschaft sowohl die Extension, als auch die Intension geben zu können, die er bräuchte, um ein spezifisches Feld menschlicher Lebens- und Handlungswelt abgrenzen und charakterisieren zu können. Es bleibt nun noch ein letzter Versuch: Der Blick auf die Disziplinen selbst - in der Hoffnung, wenigstens hier genug Einheit ausmachen zu können, um dann vielleicht eine Idee einer dann allerdings disziplinär gefärbten Wissenschaftlichen Bildung entwickeln zu können.

Tatsächlich läßt sich wohl behaupten, daß in Zeiten von durch die jeweiligen Fachgesellschaften überwachten Kerncurricula das, was eine Disziplin letztlich ausmacht, hinreichend genug bestimmt und festgelegt ist. Es sind gerade diese curricularen Rahmen, die dafür sorgen, daß die Studierenden einigermaßen ähnlich in ihre Disziplin hineinsozialisiert werden. Wie erfolgreich das ist, läßt sich an den Kommunikationsschwierigkeiten erkennen, die bereits Studierende verschiedener Disziplinen miteinander haben. Auch wenn sich natürlich spezifische Ausrichtungen der jeweiligen Disziplinen an verschiedenen Universitäten finden lassen, so erscheinen diese doch zumeist als Variationen über ein Thema. $\mathrm{Da}$ dieses auf internationaler Ebene schon wieder ganz anders aussehen kann, sei hier nur der Vollständigkeit wegen erwähnt.

\footnotetext{
${ }^{15}$ Engelen, Eva-Maria et al. (Hrsg.): Heureka - Evidenzkriterien in den Wissenschaften, Heidelberg: Spektrum Akademischer Verlag 2010.
} 
Auf einer ganz anderen, jedoch mindestens ebenso wichtigen Ebene ist die Einheit der Disziplinen in sich allerdings nicht immer, vielleicht sogar eher selten gegeben. Die Rede ist von der Identifikation der Wissenschaftler_Innen mit ihrem Gegenstand und mit ihrer eigentlichen Arbeit - dem wissenschaftlichen Tun, welches sich rein am Gegenstand ausrichtet bzw. ausrichten sollte. Es gehört wohl zu den am meisten ignorierten Tatsachen, daß die sachliche Logik, nach welcher das wissenschaftliche Arbeiten stattfindet bzw. stattfinden müßte, etwas grundlegend anderes ist als die persönliche Logik der Wissenschaftler_Innen, nach denen sie ihre Arbeit ausüben: Was gut ist für die Wissenschaft, ist noch lange nicht gut für die Wissenschaftler_Innen. Die Erfindung von Wissenschaft als erfolgsorientierter und entlohnter Erwerbsarbeit, von deren offiziell darstellbarem Erfolg das Wohl und Wehe, das alltägliche Leben der Wissenschaftler_Innen abhängt (das feste Einkommen auf einer unbefristeten Stelle), hat dazu geführt, daß die Erfolgslogik der Personen die Sachlogik des wissenschaftlichen Gegenstandes wenn nicht ersetzt, so doch weitgehend verdrängt hat; ${ }^{16}$ Wissenschaftler_Innen stehen zunehmend im Zwang, diese oft kaum vermittelbaren Seiten doch miteinander zu vermitteln. Es verwundert denn auch nicht, daß besonders der wissenschaftliche Nachwuchs in erster Linie an solchen Strategien interessiert ist, die den Erfolg im Wissenschaftssystem garantieren. Dessen Spielregeln sind weitgehend losgelöst von den Logiken des gegenstandsorientierten Arbeitens. Wie groß der Druck hier ist, zeigen die zahlreichen, in den letzten Jahren spektakulär aufgedeckten Fälschungen innerhalb des wissenschaftlichen Forschungsbetriebes und die ewigen Diskussionen darum, ob sich anhand quantifizierbarer Kriterien, die zunehmend der Beurteilung des wissenschaftlichen Erfolgs einzelner Wissenschaftler_Innen zugrundegelegt werden, tatsächlich Qualität messen läßt.

Letztlich bedeutet das: Erst dann, wenn sich bestimmte Inhalte einer sog. Wissenschaftlichen Bildung direkt und unmittelbar in erfolg-, d.h. anstellungsversprechende Strategien innerhalb des Wissenschaftsbetriebs umsetzen lassen, wird sie für die Wissenschaftler_Innen wirklich interessant. Allein eine Orientierung am wissenschaftlichen Gegenstand können sich nur diejenigen leisten, die bereits eine feste Anstellung und damit eine in gewissem Maße gesicherte Zukunft besitzen; für alle anderen bleiben solche Überlegungen eigentlich purer Luxus.

Was bedeutet das alles nun für ein Konzept Wissenschaftlicher Bildung?

4) Das Dilemma der Wissenschaftlichen Bildung

\footnotetext{
${ }^{16}$ Vgl. auch dazu den Beitrag von Tilman Reitz in diesem Band.
} 
Zunächst ist wohl offensichtlich: Wenn es die Wissenschaft nicht gibt, so kann es auch nicht die Wissenschaftliche Bildung geben. Insofern nicht davon ausgegangen werden kann, daß die einzelnen Disziplinen alle etwas gemeinsam hätten, läßt sich auch kein Bildungskonzept vorstellen, das a) inhaltlich so gefüllt wäre, daß es sich unterscheiden ließe von einem allgemeinen Bildungskonzept, welches für alle Menschen generell gültig wäre, und das b) über alle Grenzen der wissenschaftlichen Disziplinen hinweg für alle kommunizierbar und nachvollziehbar sein könnte, insofern sich die Epistemologien der Disziplinen zu sehr voneinander unterscheiden.

a) Es ließe sich zwar vielleicht sagen, daß Wissenschaftler_Innen zu ihrem Tun ein reflexives Verhältnis unterhalten sollten - doch ist das eben keine für die Wissenschaft spezifische Forderung, sondern Bestandteil einer allgemeinen Bildungsidee, insofern zu hoffen wäre, daß Menschen generell ein reflexives Verhältnis zu ihren Handlungen einnehmen - völlig unerheblich, ob es sich hierbei um Ärzt_Innen, Manager_Innen oder Sachbearbeiter_Innen handelt: Selbstreflexivität ist einfach Ausdruck einer generellen Bildung bzw. Gebildetheit. Selbst solche scheinbar im modernen Wissenschaftsbetrieb positiv bewerteten Haltungen wie die Bereitschaft zur Transund Interdisziplinarität sind, mit Blick auf den Gegenstand, lediglich Ausdruck der als allgemein anzusprechenden Forderung, sein Tun in der am meisten erfolgversprechenden Weise auszuüben und, wie man so sagt, ,über den Tellerrand zu schauen'. Mit anderen Worten: Sie sind nichts Wissenschaftstypisches. Es scheint äußerst schwierig zu sein, zwischen der Ebene einer allgemeinen Bildungstheorie und der Ebene konkreter, disziplin-bezogener Bildungsvorstellungen eine Meso-Ebene allgemeiner Wissenschaftlicher Bildung zu etablieren und ein entsprechendes Konzept zu entwerfen.

Die bereits angesprochene Trennung von Sach- und Personenlogik sorgt dazu meist für eine Kollision der verschiedenen Vorstellungen einer immer mit Blick auf den Gegenstand, auf die Wissenschaft und ihre Rolle in der Gesellschaft, formulierten Bildungsidee und den persönlichen Interessen der einzelnen Wissenschaftler_Innen. Vor allem mit der sich gerade etablierenden Praxis, solche Bildungsdebatten in den Bereich der Doktorand_Innen und Postdoktorand_Innen zu verlagern, wird dort eine Spannung erzeugt zwischen den real gefühlten Notwendigkeiten der Gestaltung einer Karriere und den zumeist als Luxus wahrgenommen Überlegungen zur sog. Wissenschaftlichen Bildung: Die Antwort darauf, wie die zusätzlichen Anstrengungen der Auseinandersetzung mit den oft behandelten Themen der wissenschaftlichen 
(eigentlich jedoch allgemeinen) Bildung der eigenen Karriere direkt nützen, können wohl nur wenige spontan und einsichtig geben.

b) Daß die Kommunikation der Notwendigkeit einer (zumeist allgemeinen) Bildung für viele Wissenschaftler_Innen schwierig ist, hat darüber hinaus noch einen weiteren Grund als allein den der Unklarheit der direkten Verwertbarkeit: Es gibt hier schlicht ein Kommunikationsproblem, welches aus der grundsätzlichen Verschiedenheit der Disziplinen resultiert.

Bildungstheorien jeglicher Art werden im Allgemeinen im Jargon der Geistes-, genauer: der geisteswissenschaftlich orientierten Bildungswissenschaften formuliert und vorgetragen - vor allem notwendigerweise dann, wenn sie normativ sind. In sehr spezifischer Sprache wird dort entwickelt und argumentiert, worin Bildung bzw. Gebildetheit eigentlich besteht. Ebenso speziell sind die Strategien und Argumente, mit denen ihre Notwendigkeit verteidigt wird. Das alles könnte für Geisteswissenschaftler_Innen noch einsichtig sein (was sich allerdings in der Realität auch nicht bewahrheitet: bildungstheoretische Debatten erscheinen auch vielen Geisteswissenschaftler_Innen, die nicht bildungsphilosophisch interessiert sind, als eher fremd). Die anderen Wissenschaften unterscheiden sich zumeist jedoch so sehr in ihrem Sprachspiel und ihrer Epistemologie, daß für sie derartige Erläuterungen und Begründungsstrategien kaum nachvollziehbar sein können. Die grundlegende Disparität der Disziplinen führt dazu, daß das, was in der einen formuliert wird, in der anderen kaum verstehbar ist. Mit anderen Worten: Die Wissenschaftliche Bildung hätte, wollte sie gesamtwissenschaftlich gelten, ein Legitimationsproblem, weil sie sich notwendigerweise in einer nur partikularen Logik und Sprache ausdrücken muß; das Universale läßt sich nicht mit nur partikular gültigen Mitteln ausdrücken und begründen. Wissenschaftliche Bildung bleibt in ihrer Theorie immer nur eingeschränkt zugänglich und nachvollziehbar - wenn sie denn tatsächlich versuchen sollte, sich mit wissenschaftlichen Mitteln vorzustellen, und nicht einfach auf Allgemeinplätze rekurriert.

Gerade der letzte Punkt erzeugt gewissermaßen ein Paradox: Wer nicht aus der epistemic culture stammt, innerhalb derer die Theorie Wissenschaftlicher Bildung formuliert wurde, sondern einer anderen, und die Theorie trotzdem versteht, gar affirmiert - der_die hat das Ziel gewissermaßen bereits erreicht, insofern sie_er bereits um die Relevanz des Überschreitens eigener Fachgrenzen weiß und wichtige Schritte auf dem Weg des Erlernens der fremden Sprache schon gegangen ist. Etwas allgemeiner formuliert, hieße das: Die Notwendigkeit 
Wissenschaftlicher Bildung erschließt sich nur denjenigen Wissenschaftler_Innen, die bereits über sie verfügen; Wissenschaftliche Bildung setzt sich selbst voraus, wenn sie sich als Bildungswissenschaft an Wissenschaftler_Innen richtet - Wissenschaftliche Bildung unterliegt so einem Zirkelschluß.

Noch einmal zur Verdeutlichung: Hier soll nicht der Sinn einer Theorie Wissenschaftlicher Bildung bezweifelt werden - der Autor der vorliegenden Zeilen zweifelt überhaupt nicht an dieser Sinnhaftigkeit, wenn deutlich gemacht wird, daß es sich eigentlich um eine Theorie der Allgemeinen Bildung handelt, die für alle gültig wäre und nicht nur für Wissenschaftler_Innen. Nur entstammt eben der hier Schreibende - wie auch der vorliegende Text - einer spezifischen wissenschaftlichen Disziplin und damit Tradition, und er weiß um diese Eingebundenheit - eine Eingebundenheit, die dazu führt, daß die vorgetragenen Argumente wahrscheinlich unzugänglich sind für diejenigen Wissenschaftler_Innen, die einer anderen Disziplin entstammen. Es ist nicht möglich, die Argumentation so zu entwerfen, daß sie von ausnahmslos allen Wissenschaftler_Innen verstanden und anerkannt werden würde - dieses bräuchte eine disziplin-unabhängige, aber immer noch wissenschaftliche Sprache und Epistemlogie. Eine solche gibt es nicht, wie sich bereits zeigte.

Dieses Problem ließe sich zukünftig umgehen, wie in einem letzten Schritt noch vorgeschlagen werden soll.

5) Zur Möglichkeit Allgemeiner Bildung in der Wissenschaft Was läßt sich nun zusammenfassend sagen? Sollte man die Idee Wissenschaftlicher Bildung, also einer Allgemein-Bildung für Wissenschaftler, generell fallenlassen, weil sie theoretische und praktische Probleme aufwirft? Sicher nicht: Das Gewahrwerden solcher Probleme kann dazu führen, sich über den Platz solcher Überlegungen genauer Gedanken zu machen.

Wie gezeigt werden konnte, verhindert gewissermaßen die disziplin-gebundene Wissenschaftlichkeit der Wissenschaftler_Innen selbst einen Zugang zur Idee der Wissenschaftlichen Bildung, die es als solche zwar nicht geben kann, die allerdings als Idee einer Allgemeinen Bildung zu reüssieren vermag. Damit stellt sich also die Frage, wie man die Wissenschaftler_Innen ganz allgemein in einen Prozeß des Sich-Bildens einbinden, in ihnen einen solchen Prozeß der Bildung initiieren und begleiten könnte. Dieses mag am ehesten dort möglich sein, wo sich weder bereits eine disziplinär gesteuerte Verengung des Denkens ereignet hat, noch schon Logiken des Erwerbs und Lebenserhalts ausgeprägt haben, die alle anderen Normative überstrahlen. Mit anderen Worten: Nicht die Doktorand_Innen oder PostDoktorand_Innen wären der adäquate Ansprechpartner für derartige Bemühungen, sondern 
die Studienanfänger bzw. Studierenden im Grund- oder Bachelor-Studium. Insofern es sich gerade nicht um eine spezifisch wissenschaftliche, sondern um eine Allgemein-Bildung handeln würde, die man anstreben müßte, wäre sie auch relevant nicht nur für diejenigen, die am Ende ,echte‘ Wissenschaftler_Innen werden: Es geht ganz generell um die Weiterführung der Bildungsprozesse an der Universität - Bildungsprozesse, die nun einen Umgang mit dem an den Universitäten Geforderten, Erlernten, Ansozialisierten ermöglichen. Der natürliche Ort einer solchen Bildung jenseits der disziplinären Grenzen ist ein Studium Generale - und es mag dann jeder Disziplin obliegen, dieses notwendig Allgemeine auf die spezifischen Probleme der jeweiligen Disziplin anzuwenden und im Graduate- und Postgraduate-Bereich zu vertiefen.

Natürlich - ein Studium Generale scheitert heutzutage zumeist an der Zeit, die man für ein Erststudium zugestanden bekommt oder sich auch nur nehmen kann (und an der Illusion der Berufsqualifizierung, die beständig aufrecht erhalten wird). Und es mag sein, daß derartige Reflexionsprozesse, die dann ein Stück weit auch relativierend auf das hehre Bild der Wissenschaft einwirken, nicht überall und immer affirmiert werden, und man deshalb darauf angewiesen ist, daß dann bereits Graduierte selbst entscheiden, daß hier eine Dimension wissenschaftlichen Arbeitens existiert - eine spezifische Form von Reflexivität etwa -, die auszuloten sich lohnt (in einem ganz anderen Sinne als dem der Karriere-Relevanz). Solange wir nicht in der besten aller möglichen Welten leben, bleibt vielleicht nur der freiwillige Zugang über die Graduierten, die entweder auf wundersame Weise bereits die ersten Schritte getan haben auf dem Bildungsweg und sich für bildungsrelevante Sachverhalte interessieren oder die man verführt mit Karriereversprechen, um ihnen unter der Hand Reflexionsangebote zu machen, die sie nicht ablehnen können. Perfekt ist das nicht ... aber es ist besser als gar nichts.

\section{Literatur}

- Engelen, Eva-Maria et al. (Hrsg.): Heureka - Evidenzkriterien in den Wissenschaften, Heidelberg: Spektrum Akademischer Verlag 2010.

- Feyerabend, Paul: Against Method [1975], London: Verso ${ }^{3} 1993$ (deutsch: Wider den Methodenzwang, Frankfurt am Main: Suhrkamp 21986).

- Fischer, Ernst Peter: Die andere Bildung - Was man von den Naturwissenschaften wissen sollte, München: Ullstein 2001. 
- Fleck, Ludwig: Entstehung und Entwicklung einer wissenschaftlichen Tatsache. Einführung in die Lehre vom Denkstil und Denkkollektiv [1935], Frankfurt am Main: Suhrkamp 1980.

- Hacking, Ian: The Disunities of the Sciences, in: Galison, Peter/ Stump, David J. (Hrsg.): The Disunity of Science: Boundaries, Contexts, and Power, Stanford: University Press 1996, S. 37-74.

- Knorr-Cetina, Karin: The Manufacture of Knowledge. An Essay on the Constructivist and Contextual Nature of Science, Oxford: Pergamon Press 1981 (deutsch: Die Fabrikation von Erkenntnis. Zur Anthropologie der Naturwissenschaft, Frankfurt am Main: Suhrkamp 1984, erweiterte Neuauflage 2002).

- Knorr-Cetina, Karin: Epistemic Cultures. How the Sciences Make Knowledge [1999], Cambridge (Mass.): Harvard University Press ${ }^{3} 2003$ (deutsch: Wissenskulturen. Ein Vergleich naturwissenschaftlicher Wissensformen, Frankfurt am Main: Suhrkamp 2002).

- Knorr-Cetina, Karin: Culture in Global Knowledge Societes: Knowledge Cultures and Epistemic Cultures, in: Interdisciplinary Science Reviews 32/4 (2007), S. 361-375.

- Kuhn, Thomas S.: The Structure of Scientific Revolutions [1962], Chicago: University of Chicago Press ${ }^{2} 1970$ (deutsch: Die Struktur wissenschaftlicher Revolutionen, Frankfurt am Main: Suhrkamp ${ }^{2}$ 1976).

- Schwanitz, Dietrich: Bildung. Alles was man wissen muß, Frankfurt am Main: Eichborn 1999.

- Serres, Michel (Hrsg.): Éléments d'histoire des sciences, Paris: Bordas 1989 (deutsch: Elemente einer Geschichte der Wissenschaften, Frankfurt: Suhrkamp 1998).

- Wittgenstein, Ludwig: Philosophische Untersuchungen, Frankfurt am Main: Suhrkamp 1953. 